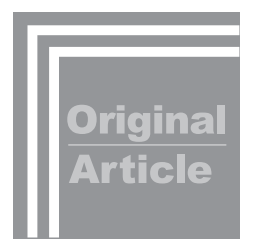

\title{
Monitoring and Protection against Radiation Dose to Eyes of Operators Performing Neuroendovascular Procedures: A Nationwide Study in Japan
}

Hajime Sakamoto, ${ }^{7}$ Takashi Moritake, ${ }^{2}$ Lue Sun, ${ }^{3}$ Ikuo Kobayashi, ${ }^{4,5}$ Satoru Kawauchi, ${ }^{6}$ Toshi Abe, ${ }^{7}$ Atsuko Tsukamoto, ${ }^{8}$ Yuh Morimoto, ${ }^{9}$ Hiroyuki Daida, ${ }^{1}$ and Yuji Matsumaru ${ }^{10}$

Objective: To meet the new standard of the annual dose limit for the eye lens recommended by the International Commission on Radiation Protection, radiation doses of neuroendovascular procedures in Japanese institutions were investigated.

Methods: Radiation doses to operators involved in 304 neuroendovascular procedures at 30 Japanese institutions were prospectively surveyed. The institutions recruited at an annual meeting of the Japanese Society for Neuroendovascular Therapy participated voluntarily. A maximum of 10 wireless dosimeters were attached to the radiation protection (RP) goggles, the ceiling-mounted RP shielding screen, and the operators' forehead and neck over the protective clothing. Doses recorded inside the goggles were defined as eye lens doses for operators who wore RP goggles, while doses to the forehead were defined as eye lens doses for those who did not. The shielding effect rates of the protection devices were calculated, and statistical analysis was performed for the comparison of radiation doses.

Results: From 296 analyzed cases, mean eye lens radiation doses per procedure were $0.088 \mathrm{mGy}$ for the left eye and $0.041 \mathrm{mGy}$ for the right eye. For the left eye, that dose without RP equipment was $0.176 \mathrm{mGy}$ and that with RP goggles plus an RP shielding screen was $0.034 \mathrm{mGy}$. Four parameters, including left eye dose, air kerma at the patient entrance reference point, fluoroscopic time, and the total number of frames, were assessed for five types of neurovascular procedures. Of them, transarterial embolization for dural arteriovenous fistula was associated with the highest eye lens dose at $0.138 \mathrm{mGy}$. The shielding effect rates of protection goggles were $60 \%$ for the left and $55 \%$ for the right RP goggle. The mean doses to the inner and outer surfaces of the RP shielding screen were $0.831 \mathrm{mGy}$ and $0.040 \mathrm{mGy}$, respectively, amounting to a shielding effect rate of $95 \%$.

Conclusion: To meet the new standard, both RP goggles and RP shielding screens are strongly recommended to be used effectively. Without proper use of radiological protection devices, the number of neuroendovascular procedures that one operator performs per year will be limited under the new guideline.

Keywords > eye lens dose, neuroendovascular, operator, radiation dose, radiation protection

\footnotetext{
${ }^{1}$ Department of Radiological Technology, Faculty of Health Science, Juntendo University, Tokyo, Japan

${ }^{2}$ Department of National Institute of Radiological Sciences, National Institutes for Quantum and Radiological Science and Technology, Chiba, Chiba, Japan

${ }^{3}$ Health and Medical Research Institute, Department of Life Science and Biotechnology, National Institute of Advanced Industrial Science and Technology (AIST), Tsukuba, Ibaraki, Japan

${ }^{4}$ Research Institute of Nuclear Engineering, University of Fukui, Tsuruga, Fukui, Japan

${ }^{5}$ Nagase Landauer Ltd., Tsukuba, Ibaraki, Japan

${ }^{6}$ Department of Radiology, Toranomon Hospital, Tokyo, Japan

${ }^{7}$ Department of Radiobiology, Kurume University School of Medicine, Kurume, Fukuoka, Japan

${ }^{8}$ Department of Radiology, NTT Medical Center, Tokyo, Japan
}

\begin{abstract}
${ }^{9}$ Faculty of Health Science, Juntendo University, Tokyo, Japan ${ }^{10}$ Division of Stroke Prevention and Treatment, Department of Neurosurgery, Faculty of Medicine, University of Tsukuba, Tsukuba, Ibaraki, Japan
\end{abstract}

Received: September 3, 2021; Accepted: October 25, 2021 Corresponding author: Hajime Sakamoto. Faculty of Health Science, Department of Radiological Technology, Juntendo University, 2-1-1, Hongo, Bunkyo-ku, Tokyo 113-8421, Japan Email: h.sakamoto.qv@juntendo.ac.jp

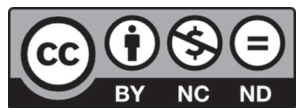
This work is licensed under a Creative Commons Attribution-NonCommercialNoDerivatives International License.

(C)2021 The Japanese Society for Neuroendovascular Therapy 


\section{Introduction}

Modern interventional radiology (IR) has been evolving for over 50 years. ${ }^{1}$ Since its advent, neuroendovascular procedures have developed in diagnosing and treating diseases. ${ }^{2)}$ However, neuroendovascular procedures frequently involve long fluoroscopic time, high-dose fluoroscopic mode, and greater numbers of exposure sessions, which increase radiation dosage for patients and operators, resulting in radiation injuries. ${ }^{3-7)}$ We previously reported that patient skin doses from neuroendovascular procedures are higher than those from abdominal and cardiac IR. ${ }^{8)}$ Specialized skills are required to safely perform neuroendovascular procedures, leading the radiation risk to increase to some particular operators. ${ }^{9)}$

In 2011, the International Commission on Radiation Protection (ICRP) recommended reducing the dose limit for the eye lens from 150 to $20 \mathrm{mSv}$ per year, averaged over five years, with no annual dose in a single year exceeding $50 \mathrm{mSv} .{ }^{10)}$ The reduction has had a significant impact on healthcare professionals in many aspects. ${ }^{11)}$ In April 2021, Japanese law was revised to meet the ICRP new standard.

In this study, we investigated eye lens radiation doses among neuroendovascular operators at 30 representative hospitals performing high numbers of annual neuroendovascular procedures across Japan. This is the first nationwide, large-scale study of eye lens doses incurred by healthcare professionals performing neuroendovascular procedures in Japan.

\section{Materials and Methods}

\section{Data collection}

Radiation doses to operators involved in 304 neuroendovascular procedures from 30 Japanese institutions were surveyed in 2014. Participating operators were recruited during the 29th annual meeting of the Japanese Society for Neuroendovascular Therapy in 2013. A set of 10 dosimeters was sent to each institution to measure the radiation dose to operators for each procedure. The set of dosimeters was returned for analysis after each procedure. This process was repeated multiple times to record radiation doses experienced by operators performing neuroendovascular procedures among 30 institutions. A total of 304 neuroendovascular procedures were prospectively surveyed. The neuroendovascular procedures performed included coil embolization of cerebral aneurysms (coil TAE; 167 cases), carotid artery stenting
Table 1 Baseline data and acronyms

\begin{tabular}{lc} 
Variable & Cases \\
Patients' characteristics & \\
Male & 136 \\
Female & 158 \\
Unknown & 10 \\
Ages & 62.8 \\
& (mean SD 14.9) \\
Surgery & \\
Coil TAE & 167 \\
CAS & 46 \\
TAV · TAE of dAVF & 25 \\
AVM TAE & 14 \\
Tumor TAE & 14 \\
Others & 38 \\
Total & 304 \\
Questionnaire and forms & \\
Total & 304 \\
Number of valid responses & 296 \\
Protective devices used during the operation & \\
Goggle · screen & 78 \\
Goggle & 23 \\
Screen & 124 \\
None & 71 \\
Total & 296 \\
\hline AVM TAE: transarterial embolization for an arteriovenous malformation; \\
CAS: carotid artery stenting; Coil TAE: coil embolization of cerebral aneu- \\
rysms; dAVF TAE-TVE: transvenous embolization or transarterial emboliza- \\
tion for a dural arteriovenous fistula; SD: standard deviation; Tumor TAE: \\
tumor transarterial embolization \\
\end{tabular}

(CAS; 46), transvenous or transarterial embolization for a dural arteriovenous fistula (dAVF TAE-TVE; 25), transarterial embolization for an arteriovenous malformation (AVM TAE; 14), Tumor TAE (14), and other (38) (Table 1). Excluding eight that did not complete the questionnaire, responses of 296 were analyzed in this study. The use of radiation protection (RP) equipment and the gender and ages of the patients are shown in Table 1. Fourteen out of 30 institutions used $0.07 \mathrm{~mm}$ Pb-type (Panorama Shield; Toray, Tokyo, Japan) RP goggles, and one institution used $0.75 \mathrm{~mm}$ $\mathrm{Pb}$-type RP goggles. The rest of the 15 institutions did not use RP goggles. In this study, we included only $0.07 \mathrm{~mm}$ Pb-type RP goggles.

\section{Measurement of radiation doses for neuroendovascular operators}

NanoDot dosimeters (Nagase-Landauer, Ibaraki, Japan) were used to measure radiation doses (air-kerma) among neuroendovascular operators. As shown in Table 2 and Fig. 1, between four to 10 of these wireless and radiolucent $1 \mathrm{~cm} \times 1 \mathrm{~cm}$ dosimeters were attached to the RP goggles, the ceiling-mounted RP shielding screen, and the operators' forehead and neck. Because it is infeasible to 
Table 2 Quantity and locations of dosimeters and RP devices

\begin{tabular}{cccccc} 
& \multicolumn{5}{c}{ Numbers of dosimeters } \\
\cline { 2 - 6 } Method A & Goggle & Board & Forehead & Neck & Total \\
Method B & 6 & 2 & 1 & 1 & 10 \\
Method C & - & - & 1 & 1 & 8 \\
Method D & - & 2 & 3 & 1 & 6 \\
\hline
\end{tabular}

Operators of method A used both RP goggles and a screen, those of method B used only RP goggles, those of method $C$ used a RP screen only, and those of method D used neither RP goggles nor a screen. RP: radiation protection
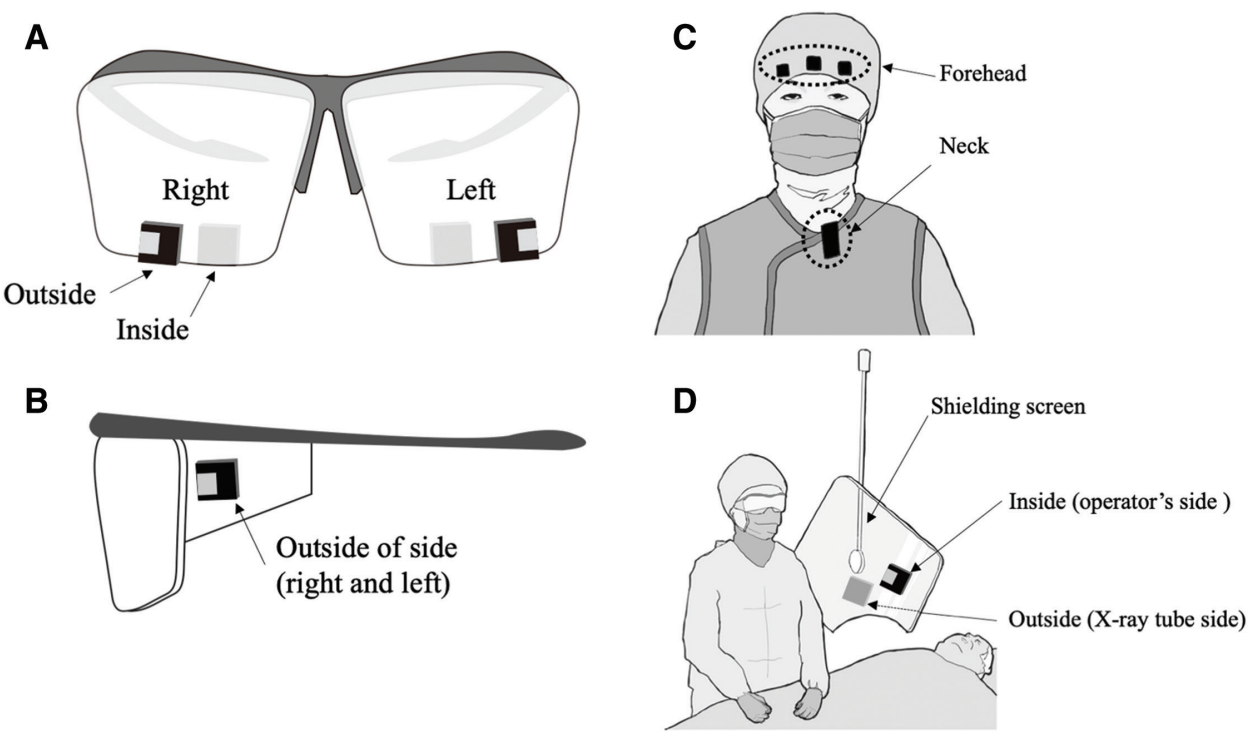

Fig. 1 NanoDot dosimeters located at four sites. NanoDot dosimeters placed in four positions. (A and B) With RP goggles (6 dosimeters) (C) and without, with three on the forehead; and (D) regardless of goggles, anterior and posterior of the RP shielding screen. RP: radiation protection

measure actual eye lens doses in vivo, the doses inside the RP goggles were defined as eye lens doses. Radiation doses obtained by dosimeters placed on the left and right sides of the forehead were defined as the eye lens doses for operators who did not wear RP goggles.

\section{Questionnaire}

The operators were asked to complete a form and a questionnaire. Data regarding operations were collected, including the type of neuroendovascular procedures and equipment, where they stood in relation to other staff and the X-ray tube (nearest the tube or second or other position), type of personal protection equipment used, dose metrics as provided by the equipment, fluoroscopic time, and $\mathrm{C}$-arm angle (working angle).

\section{Calculation of shielding factors}

The shielding effect rates of the RP goggles and the ceiling-mounted RP shielding screen were calculated by comparing the radiation doses obtained by the nanoDots placed on either side of the surfaces of the devices.

\section{Statistical analysis}

The Mann-Whitney U test was used for pairwise comparison of radiation doses. For comparison of bilateral lens doses, the Wilcoxon signed-rank test was used.

\section{Ethics approval}

The research within our submission has been approved by the ethics institutional review board of University of Yamanashi (2014/01/31 1153).

\section{Results}

\section{Radiation doses during neuroendovascular procedures under various conditions}

Table 1 summarizes the RP devices used during neuroendovascular procedures, which included $0.07 \mathrm{~mm}$ Pb-type 


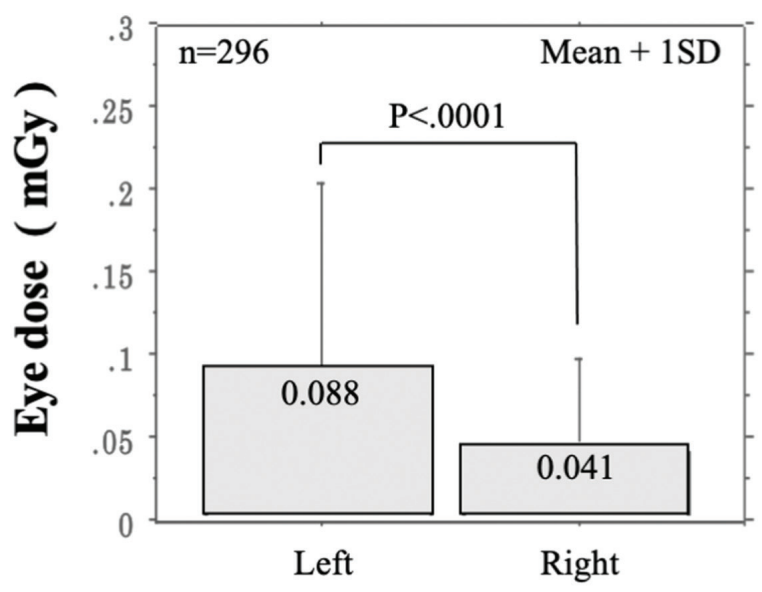

Fig. 2 The mean value of the left was significantly higher than that of the right eye dose: comparison of bilateral eye dose. The $P$ value was determined using the Wilcoxon signedrank test.

Table 3 Summary of radiation doses in neurovascular procedures

\begin{tabular}{lcc} 
& Cases (296) & $\begin{array}{c}\text { Lens dose, mean (SD) } \\
\text { (mGy) }\end{array}$ \\
$\begin{array}{l}\text { Operator's lens dose } \\
\text { Left eye }\end{array}$ & $0.088(0.115)$ \\
Right eye & & $0.041(0.057)$ \\
Left lens dose and position of operator & $201(68 \%)$ & $0.097(0.120)$ \\
Closest to X-ray tube & $95(32 \%)$ & $0.066(0.091)$ \\
Second or third closest & & \\
Left lens dose with protective devices & 78 & $0.034(0.049)$ \\
Goggles · screen & 23 & $0.055(0.052)$ \\
Goggles & 124 & $0.080(0.102)$ \\
Screen & 71 & $0.176(0.152)$ \\
None & 101 & \\
Comparison between inside and outside of the protective devices & $0.038(0.051)$ \\
Left eye goggle inside & 101 & $0.095(0.147)$ \\
Left eye goggle outside & 101 & $0.029(0.053)$ \\
Right eye goggle inside & 101 & $0.064(0.091)$ \\
Right eye goggle outside & 202 & $0.040(0.050)$ \\
Screen inside & 202 & $0.831(0.780)$ \\
Screen outside & & \\
\hline SD: standard deviation & &
\end{tabular}

RP goggles with an RP shielding screen (78 cases), RP goggles without an RP shielding screen (23), an RP shielding screen without goggles (124), and neither (71). The radiation doses of $0.07 \mathrm{~mm} \mathrm{~Pb}$-type $\mathrm{RP}$ goggles were analyzed. Mean doses were $0.088 \mathrm{mGy}$ and $0.041 \mathrm{mGy}$ to the left and right eyes, respectively (Fig. 2).

Among 296 operators, 201 (68\%) reported standing closest to the X-ray tube (1st operator), and the other 95 $(32 \%)$ reported standing second or third closest (2nd or 3rd operators) or switching positions during the procedure. The mean left eye dose of the operators who remained standing closest to the X-ray tube was $0.097 \mathrm{mGy}$ and that of the operators standing second or third closest or in switching positions during the procedure was $0.066 \mathrm{mGy}$ (Table 3).
The mean radiation dose of the left eyes of operators that used neither RP goggles nor an RP shielding screen was $0.176 \mathrm{mGy}$ and that of those who used both was $0.034 \mathrm{mGy}$. That of those who used RP goggles only was $0.055 \mathrm{mGy}$ and that of those who used an RP shielding screen only was 0.080 mGy (Fig. 3). The mean doses to the inner and outer surfaces of the left RP goggle were $0.038 \mathrm{mGy}$ and $0.095 \mathrm{mGy}$, respectively, and those to the right RP goggle were 0.029 mGy and $0.064 \mathrm{mGy}$, respectively (Table 3). Based on these values, the shielding effect rates were $60 \%$ for the left and $55 \%$ for the right RP goggle. The mean doses to the inner and outer surfaces of the RP shielding screen were $0.831 \mathrm{mGy}$ and $0.040 \mathrm{mGy}$, respectively, amounting to a shielding effect rate of $95 \%$ for the RP shielding screen (Table 3 ). 


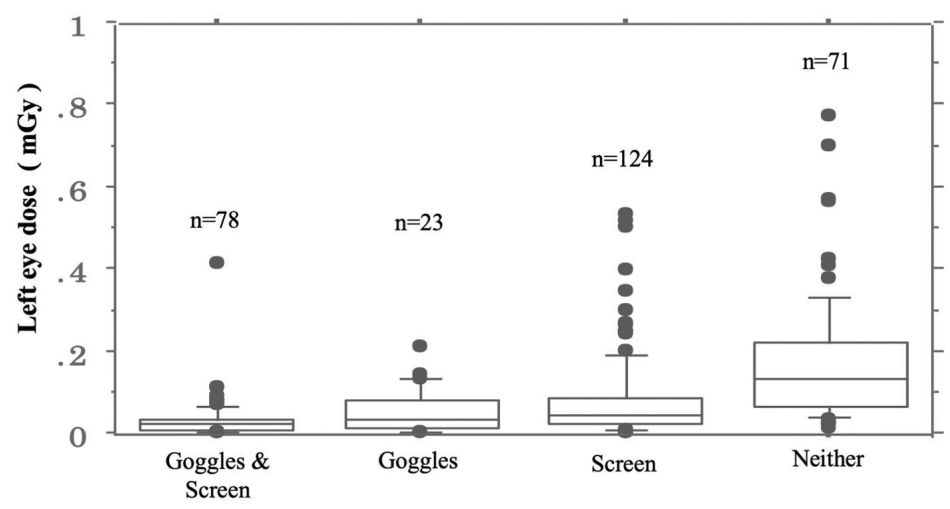

Fig. 3 Comparison of mean dose of left eye using RP goggles and a ceiling-mounted RP shielding screen. RP: radiation protection

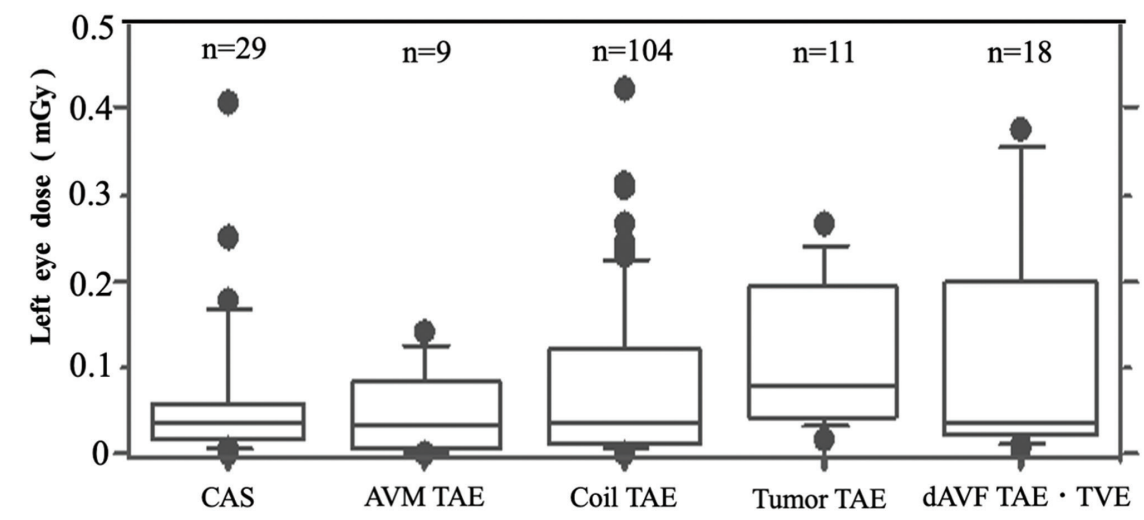

\begin{tabular}{|c|c|c|c|c|c|}
\hline & CAS & AVM TAE & Coil TAE & Tumor TAE & dAVF TAE $\cdot$ TVE \\
\hline Left eye dose (mGy) & $0.068(0.088)$ & $0.054(0.050)$ & $0.087(0.106)$ & $0.117(0.087)$ & $0.138(0.195)$ \\
\hline Ka,r* (mGy) & $915.3(748.9)$ & $2394.1(1161.7)$ & $2682.7(2013.4)$ & $1187.2(651.6)$ & $3109.9(1489.0)$ \\
\hline Fluoroscopic time (min) & $38.1(26.7)$ & $95.4(65.0)$ & $1187.2(651.6)$ & $38.2(18.4)$ & $121.7(48.9)$ \\
\hline Total frame numbers & $953(759)$ & $1490(122)$ & $3110(1489.0)$ & $1225(660)$ & $3231(1508)$ \\
\hline
\end{tabular}

*Ka,r, Air-kerma at the patient entrance reference point (incident dose displayed on the equipment).

$\operatorname{Mean}(\mathrm{SD})$

Fig. 4 Left eye dose, indication dose of equipment, fluoroscopic time, and total number of frames in five neuroendovascular procedures. Comparison of left eye doses among five neuroendovascular procedures: Coil TAE, CAS, dAVF TAE-TVE, AVM TAE (14), and Tumor TAE. *Ka,r: air kerma at the patient entrance reference point (incident dose displayed on the equipment). AVM TAE: transarterial embolization for an arteriovenous malformation; CAS: carotid artery stenting; Coil TAE: coil embolization of cerebral aneurysms; dAVF TAE-TVE: transvenous embolization or transarterial embolization for a dural arteriovenous fistula; Tumor TAE: tumor transarterial embolization

\section{Comparison of left eye dose by type of neuroendovascular procedures}

We examined radiation dose differences among five types of procedures, CAS, AVM TAE, Coil TAE, Tumor TAE, and dAVF TAE·TVE. The left eye radiation doses of the 1st operators were analyzed $(n=171)$. Among them, dAVF TAE-TVE showed the highest radiation dose with a mean value of 0.138 mGy, followed by Tumor TAE ( $0.117 \mathrm{mGy})$, Coil TAE $(0.087$ mGy), CAS (0.068 mGy), and AVM TAE (0.054 mGy). The highest Kar (air-kerma at the patient entrance reference point of incident dose displayed on the equipment) was observed at 3109.9 mGy, followed by Coil TAE (2682.7 mGy), AVM
TAE (2394.1 mGy), Tumor TAE (1187.2 mGy), and CAS (915.3 mGy). The mean fluoroscopic times of dAVF TAE.TVE, AVM TAE, Coil TAE, Tumor TAE, and CAS, were $121.7 \mathrm{~min}, 95.4 \mathrm{~min}, 67.8 \mathrm{~min}, 38.2 \mathrm{~min}$, and $38.1 \mathrm{~min}$, respectively, and the total number of frames for dAVF TAE·TVE, Coil TAE, AVM TAE, Tumor TAE, and CAS were 3231, 3110, 1490, 1225, and 953, respectively (Fig. 4).

\section{Discussion}

The IR procedure is a beneficial tool for neuroendovascular operations; however, it increases the risk of injury to 
operators from radiation. ${ }^{5-7,12)}$ Neuroendovascular procedures are complicated, and for this reason, the number of digital subtraction angiography increases, leading to a longer fluoroscopic exposure time. ${ }^{13)}$ There are several types of neuroendovascular procedures. In this study, five major procedures were reported. The longest fluoroscopic time and the largest total number of frames were associated with dAVF TAE-TVE; thus, radiation dose received by operators was the highest in this procedure among the five. Eye dose generally increases with indication dose, fluoroscopic time, and the total number of frames.

The mean eye lens dose among Tumor TAE procedures was the second highest, although the indication dose, fluoroscopic time, and total number of frames in this procedure were the second lowest among the five. It is important to note that the operators of Tumor TAE in this study had a low usage rate of RP devices. Moreover, the Tumor TAE procedure irradiates a greater area than the other four, leading to a higher scatter dose. Thus, the radiation dose received by operators was higher.

During neuroendovascular procedures, operators stand to the right side of the patient. In such cases, the left side of the operator's body is more exposed to the X-ray tube. In the present study, we demonstrated that the mean dose of the left eye was 2.25 -fold greater than that of the right. For that reason, we propose that measuring left eye lens doses is essential to evaluate the degree of the operators' eye lens dose.

Our results indicate that using both RP goggles and an RP shielding screen together is very effective for reducing the risk of radiation exposure. Thus, we highly recommend the use of both RP goggles and an RP shielding screen. The shielding effects of $0.07 \mathrm{~mm} \mathrm{~Pb} \mathrm{RP} \mathrm{goggles} \mathrm{were} \mathrm{found} \mathrm{to} \mathrm{be} 60 \%$ for left eyes and $55 \%$ for right eyes. These values are consistent with those of a previous report. ${ }^{14)}$ Moreover, the shielding effect of an RP shielding screen was as high as $95 \%$. Roguin et al. reported a disproportionate number of tumors on the left side of the head and neck among IR operators. ${ }^{15)}$ Since RP goggles only protect the eyes, it is important to use an RP shielding screen that protects the entire head and neck.

The effective use of an RP shielding screen is another concern. The mean dose of the left eyes of operators who used a shielding screen ( $0.080 \mathrm{mGy})$ was approximately half of those with neither RP goggles nor RP shielding screen $(0.176 \mathrm{mGy})$, and the shielding effect rate calculated from those values is $54.5 \%$. Since the anticipated shielding effect rate of an RP shielding screen is $95 \%$, $0.080 \mathrm{mGy}$ is higher than the value expected. Types and difficulty levels of procedures may have differed between the operators who used an RP shielding screen and those who did not, which may have influenced the left eye doses. However, the question still remains on the substantial gap between the theoretical shielding effect rate and the rate obtained in this study. This suggests that the RP shielding screen might not have been used effectively. Further studies are warranted to assess best practices for the usage of an RP shielding screen.

The average dose per case in the left eye of the neuroendovascular operators without RP devices in the present study was $0.176 \mathrm{mGy}$. Converted to a $3 \mathrm{~mm}$ dose equivalent, $\mathrm{Hp}(3)$, to evaluate exposure to the eye lens, it is 0.225 $\mathrm{mSv}$ (conversion coefficient $\left[\mathrm{Sv} \mathrm{Gy}^{-1}\right.$ ] of air kerma to $\mathrm{Hp}(3)$ used 1.28). ${ }^{16)}$ The previous cumulated dose limit of 150 $\mathrm{mSv} / \mathrm{year}$ allowed individual operators to perform 470 neuroendovascular procedures per year without using any RP devices $(0.225 \mathrm{mSv} /$ procedure). To follow the new ICRP statement and to ensure an annual cumulated exposure rate of under $20 \mathrm{mSv}$, an individual operator without RP devices may perform only 89 procedures per year $(0.225 \mathrm{mSv} /$ procedure). Using RP goggles allows 286 procedures per year $(0.070 \mathrm{mSv} /$ procedure $)$ and 455 if both RP goggles and an RP board are used $(0.044 \mathrm{mSv} /$ procedure). To comply with the new dose limit, the use of RP devices is essential to safeguard the health of operators; otherwise, hospitals would need to drastically reduce the annual number of procedures performed by neuroendovascular operators.

Currently, in accordance with Japanese law, a male operator's radiation exposure dose is measured by a device worn above the neck outside radiation protective clothing and one worn on the chest inside radiation protective clothing (or at the abdomen inside protection clothing for females). The dosimeter located at the neck outside protective clothing yields a higher exposure dose than that to the eye when wearing RP goggles. To adhere to the annual lens dose limit and safely maintain the current number of procedures performed by each operator, further studies are expected to evaluate accurate radiation doses received by operator's eye lens when performing routine measurements.

\section{Limitations}

The limitations of this study include difference in the locations of dosimeters attached to obtain eye lens doses since assumed eye lens doses of operators with RP goggles were obtained from the dosimeter attached inside of the RP goggles, whereas those of operators without RP goggles were obtained by the dosimeter attached to the forehead. 


\section{Conclusion}

Since eye lens doses among operators preforming neuroendovascular procedures are considerably high, both RP goggles and an RP shielding screen are strongly recommended to be used effectively. To comply with national regulations of eye lens doses without proper use of RP devices, the number of neuroendovascular procedures that one operator can perform per year will be reduced. To safely perform the procedures, further studies are warranted to assess best practices for the usage of an RP shielding screen.

\section{Acknowledgment}

We would like to thank the following hospitals for their cooperation in this research: Kohnan Hospital, Chiba Emergency Medical Center, Sendai Medical Center, Geriatrics Research Institute and Hospital, National Cerebral and Cardiovascular Center Hospital, Toyama University Hospital, Kokura Memorial Hospital, Kurume University Hospital, Shinshu University Hospital, Juntendo University Hospital, Tokushima University Hospital, Kameda Medical Center, Kagawa University Hospital, Osaka City General Hospital, Wakayama Rosai Hospital, Dokkyo Medical University Saitama Medical Center, Nagoya University Hospital, Okayama University Hospital, Tokyo Medical and Dental University Medical Hospital, Niigata University Medical \& Dental Hospital, Kobe City Medical Center General Hospital, Kyoto University Hospital, Takeda Hospital, The Hospital of Hyogo College of Medicine, Tokushima Red Cross Hospital, The Jikei University Hospital, Toranomon Hospital, NTT Medical Center Tokyo, University of Yamanashi Hospital, and Nagasaki University Hospital.

\section{Funding}

This study was supported by the Japanese Society for Neuroendovascular Therapy.

\section{Disclosure Statement}

The author Ikuo Kobayashi is an employee of NagaseLandauer, Ltd. To maintain independence, this author was not directly involved in data collection or analysis in this study. The other authors have no conflicts of interest.

\section{References}

1) Baum RA, Baum S. Interventional radiology: a half century of innovation. Radiology 2014; 273: S75-S91.
2) Arnold MJ, Keung JJ, McCarragher B. Interventional radiology: indications and best practices. Am Fam Physician 2019; 99: 547-556.

3) Efstathopoulos EP, Pantos I, Andreou M, et al. Occupational radiation doses to the extremities and the eyes in interventional radiology and cardiology procedures. $\mathrm{Br} J$ Radiol 2011; 84: 70-77.

4) Sakamoto H, Nakamura O, Yuge M, et al. A study of patient's dose control using an area exposure product meter. Jpn J Radiol Technol 2000; 56: 1256-1265. (in Japanese)

5) Koenig TR, Wolff D, Mettler FA, et al. Skin injuries from fluoroscopically guided procedures: part 1, characteristics of radiation injury. AJR Am J Roentgenol 2001; 177: $3-11$

6) Koenig TR, Mettler FA, Wagner LK. Skin injuries from fluoroscopically guided procedures: part 2, review of 73 cases and recommendations for minimizing dose delivered to patient. AJR Am J Roentgenol 2001; 177: 13-20.

7) Vañó E, González L, Beneytez F, et al. Lens injuries induced by occupational exposure in non-optimized interventional radiology laboratories. Br J Radiol 1998; 71: 728-733.

8) Sakamoto H, Fukasawa M, Takeda $M$, et al. Exposure doses for the operator and patient in blood access intervention therapy (BAIVT). J Jpn Soc Dial Ther 2003; 36: 1199-1205. (in Japanese)

9) Sakamoto H, Ikegawa H, Kobayashi H, et al. [A study of operator's hand and finger exposure dose reduction during angiographic procedures]. Nihon Hoshasen Gijutsu Gakkai Zasshi 2009; 65: 25-34.

10) Stewart FA, Akleyev AV, Hauer-Jensen M, et al. ICRP publication 118: ICRP statement on tissue reactions and early and late effects of radiation in normal tissues and organsthreshold doses for tissue reactions in a radiation protection context. Ann ICRP 2012; 41: 1-322.

11) D'Avino V, Angrisani L, La Verde G, et al. New eye lens dose limit: status of knowledge in Campania hospitals. Int J Environ Res Public Health 2019; 16: 3450.

12) Valentin J. Avoidance of radiation injuries from medical interventional procedures: ICRP publication 85. Ann ICRP 2000; 30: 7-67.

13) Kato $M$, Chida $K$, Ishida $T$, et al. Occupational radiation exposure of the eye in neurovascular interventional physician. Radiat Prot Dosimetry 2019; 185: 151-156.

14) Haga $Y$, Chida K, Kaga $Y$, et al. Occupational eye dose in interventional cardiology procedures. Sci Rep 2017; 7: 569.

15) Roguin A, Goldstein J, Bar O, et al. Brain and neck tumors among physicians performing interventional procedures. Am J Cardiol 2013; 111: 1368-1372.

16) Behrens R. Air kerma to $\operatorname{Hp}(3)$ conversion coefficients for a new cylinder phantom for photon reference radiation qualities. Radiat Prot Dosimetry 2012; 151: 450-455. 